\title{
EL GENERAL DE SANIDAD MILITAR EZEQUIEL ABENTE Y LAGO (1841-1923) *
}

\section{THE GENERAL OF THE MEDICAL CORPS EZEQUIEL ABENTE Y LAGO (1841-1923)}

\author{
FELIPE VALDÉS HANSEN
}

\begin{abstract}
Resumen
Biografía del médico del Cuerpo de Sanidad Militar Ezequiel Abente y Lago (1841-1923). Tras estudiar la carrera de medicina en la Universidad de Santiago, ingresó como ayudante médico en el ejército donde ascendió hasta ser el primero de su escala. Fue un laureado militar por su labor en tiempos de guerra y paz. En 1996 el antiguo Hospital Militar de A Coruña fue rebautizado con su nombre, reconociendo su competente dirección del mismo durante la repatriación de los soldados de Cuba, tras perder la guerra de 1898 con los EE.UU.
\end{abstract}

\section{Palabras clave}

Biografía, Ezequiel Abente Lago, Médico, Cuerpo de Sanidad Militar, Ejército de España.

\begin{abstract}
Biography of the Spanish Army doctor Ezequiel Abente y Lago (1841-1923). After completing his medical career at the University of Santiago de Compostela, he entered the medical corps of the Spanish Army as an assistant doctor, and he was repeatedly promoted while there until he finally attained the top position in the service. He was awarded several medals in acknowledgement for his medical merits both in war and peace periods. The former Military Hospital of Coruña was officially given his name in the year 1996, thus acknowledging his competent directorship of the hospital in the period of the repatriation from Cuba of wounded soldiers after the Spanish-American war of 1898.
\end{abstract}

\section{Key Words}

Biography, Ezequiel Abente Lago, Doctor, Medical Corps, Spanish Army.

* Fecha de entrega: 5-5-2008. 
Ezequiel Abente y Lago nació en la villa coruñesa de Corcubión el 3 de abril de 1841, no muy lejos de Muxía, donde vivía la familia y ejercía como médico su padre Leandro Abente Chans, del cual heredó la vocación por la medicina. Muy cerca de allí el abuelo y bisabuelo de Ezequiel habían sido administradores de los alfolíes y las rentas reales de la villa de Laxe. No obstante, la cuna familiar de los Abente se localiza en la pequeña aldea de Allones o Anllóns, cercana a todas estas localidades costeras, aunque retirada hacia el interior. En este punto, a orillas del río de igual nombre, comienza y se desvanece el rastro de tan excepcional apellido que podemos remontar hasta los comienzos del siglo XVIII o finales del anterior. Posiblemente, tiene su origen en la galleguización o castellanización de un apellido extranjero del norte de Europa (Abent). Sin embargo, su rareza no fue óbice para que se extendiese rápidamente por estas localidades costeras, llegando hasta Coruña, Madrid y Sudamérica (Paraguay, Uruguay y Argentina), donde se conserva como primer apellido, al contrario que en Galicia donde ha desaparecido como tal en la actualidad.

El abuelo de Ezequiel, Isidro Abente Vales, se había casado con Rosario Chans Mourelle, sobrina del navegante y descubridor Francisco Antonio Mourelle de la Rúa. Con ella Isidro tuvo cuatro hijos, incluyendo el padre de Ezequiel, y cinco hijas, siendo una de ellas la madre del poeta Eduardo Pondal Abente ${ }^{1}$. Ésta y la siguiente generación de los Abente emparentaron con familias de renombre de la zona, caso de los Pondal, consolidando así una cómoda posición socio-económica. En este contexto favorable los hubo que destacaron en el campo de las letras, como el citado Eduardo, Gonzalo López Abente o Victorino Abente y Lago, primo, sobrino y hermano de Ezequiel respectivamente. Dos de ellos también despuntaron en la medicina y la ciencia. Es el caso de su primo y médico Isidro Pondal Abente, prestigioso director del balneario de Mondariz durante la belle époque, y del geoquímico Isidro Parga Pondal, nieto del anterior y uno de los científicos más importantes de Galicia.

Ezequiel tuvo seis hermanas ${ }^{2}$ y tres hermanos de rítmico nombre: Víctor, Victorino y Victoriano. Este último, al igual que Ezequiel y el padre de ambos, estudió medicina en la Universidad de Santiago, aunque después siguió el camino de su hermano Victorino que había emigrado al Paraguay. Allí ambos hermanos y sus descendientes alcanzaron prestigio social, cultural y éxito profesional. Por su parte,

\footnotetext{
${ }^{1}$ Entre la descendencia del matrimonio Abente Chans, además de Leandro, padre de Ezequiel, podemos citar al párroco Eduardo, el cual inició en la poesía a su sobrino Eduardo Pondal, hijo de su hermana Ángela Fernanda, casada con Juan Pondal Frois. Otra hija de los Abente Chans (Juana), casada también con un Pondal (José Pondal del Pozo), fue la madre del médico Isidro Pondal Abente y la bisabuela del científico Isidro Parga Pondal.
} 
Ezequiel estudió la carrera de medicina entre 1857 y 1863, obteniendo la calificación de sobresaliente en casi todas las asignaturas ${ }^{3}$. Curiosamente, el hijo coincidió en la carrera con su padre Leandro que, ya con 50 años, se matriculó en el curso de 1859-60 para completar su licenciatura en Medicina, obtenida en 1831, con la de Cirugía 4 .

Tras licenciarse Ezequiel ingresó por oposición en el Cuerpo de Sanidad Militar en 1864, como segundo Ayudante médico del Batallón de Cazadores de Tarifa, destinado en Burgos, y del enclave militar del Peñón de Vélez de la Gomera, en la costa africana 5 . En el Archivo General Militar de Segovia se conserva el expediente militar de Ezequiel que nos permite conocer pormenorizadamente su trayectoria profesional y constituye la principal fuente de información de esta biografía. Paradójicamente, esta es la razón por la que preferimos evitar la constante reiteración de las citas de dicho archivo, pues habría que añadir la consiguiente nota al final de casi todas las frases del artículo.

A finales de 1867 asciende, por antigüedad, a primer Ayudante médico y, tras quedar en situación de reemplazo, fue destinado al año siguiente al Regimiento de Infantería de Galicia en Palma de Mallorca. En la equiparación de mandos con los oficiales de los cuerpos armados ello equivalía a un ascenso de teniente a capitán ${ }^{6}$. Después, por la gracia general tras el cambio de régimen y exilio de los borbones en 1868, alcanzó el grado de Médico mayor (primer comandante), sirviendo en los regimientos de infantería de Iberia (1869-70) y Valencia (1870) en Palma, Melilla, Alhucemas y la provincia de Málaga. Es entonces cuando conoce y se casa en Málaga (1869) con la hija de un teniente de infantería: Rafaela Escobar López. Con ella tendrá siete hijos de los cuales sólo dos alcanzarán la plena madurez: una hija nacida en Vitoria, durante la guerra contra los carlistas, y el más pequeño, bautizado con el nombre del padre ${ }^{7}$. Después, a finales de 1870, pasa al Regimiento de Infantería de Cuenca, destinado en Ourense y Ferrol.

\footnotetext{
${ }^{2}$ Una de sus hermanas (Eduarda) se casó con el Director general de Aduanas José Valdés Díaz y otra (Julia) fue la madre del poeta Gonzalo López Abente.

${ }^{3}$ Archivo Histórico Universitario de Santiago de Compostela (AHUS), Universidad, Expedientes personales, leg. $5, \mathrm{n}^{\circ} .17$.

${ }^{4}$ Ibíd.

${ }^{5}$ Archivo General Militar de Segovia (AGMS), Expedientes personales, Abente Lago, Ezequiel.

${ }^{6}$ MASSONS, José María, Historia de la sanidad militar española 4, Barcelona 1994, 288.

${ }^{7}$ Del primer matrimonio alcanzaron la edad adulta Flora y Ezequiel, muriendo durante la infancia o juventud Eulogia, Engracia, Eduarda, Elena y Julio. Del segundo matrimonio sobrevivió Leopoldo y falleció durante la infancia el primogénito Narciso. Archivo de la Real Academia Galega (ARAG), Fondo Ezequiel Abente y Lago, 33/1.
} 
En 1872, cuando el pretendiente al trono de España Carlos VII cruza la frontera hispano-francesa con la determinación de iniciar otra guerra carlista, la tercera, se inicia un período especialmente convulso en la vida de Ezequiel. En mayo de ese año parte del Ferrol con su batallón hacia San Sebastián para incorporarse al Ejército de Operaciones del Norte. Una vez allí, fue de los primeros en salir en campaña e inaugurar las hostilidades, pues el 18 de mayo asiste al primer enfrentamiento de importancia, el de Arrigorriaga (Vizcaya), tras las escaramuzas iniciales. Por su actuación allí fue condecorado con la Cruz de primera clase del Mérito Militar con el distintivo rojo reservado a los hechos de armas. Tras acompañar al batallón en todos sus movimientos regresa al Ferrol. En este aparente tranquilo destino le sorprende la insurrección republicana del

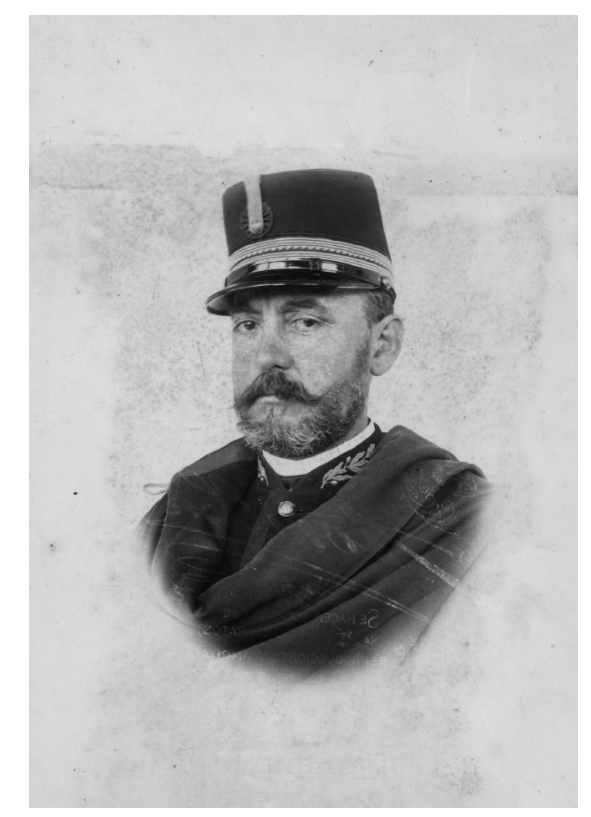

Ezequiel Abente y Lago (1841-1923). Archivo de la Real Academia Galega (ARAG).

Arsenal, la cual ayuda a sofocar entre el 15 y 17 de octubre de 1872. Por esta otra acción recibirá la Cruz de primera clase del Mérito Naval, también con distintivo rojo, además de ser recompensado con el grado honorífico de Subinspector médico de segunda clase que le fue permutado por el empleo de Médico mayor por mérito de guerra.

Después del ascenso queda en situación de remplazo en espera de destino, durante los primeros meses de 1873 que disfruta en la casa familiar de Muxía. La paz y el reposo terminan con su vuelta al Ejército del Norte, incorporado ahora a la Columna de 
Operaciones del Gobernador de Vizcaya, en Bilbao, desde el mes de marzo hasta julio. De allí pasa al Regimiento de Infantería de Almansa que, operando en el centro, interviene en la acción de Palomar el 14 de septiembre. En el verano de 1874, otra vez con el Ejército del Norte y formando parte del Regimiento de Caballería de Talavera, participa en los combates de Estella (Navarra) del 25 al 28 de junio y de Oteiza (Navarra) el 11 de agosto. Después es destinado al Regimiento de Ingenieros en Miranda del Ebro (Burgos), con el que asiste al socorro del sitio de Irún (Guipúzcoa) entre el 10 y 12 de noviembre. Por estos hechos de armas, a finales de ese mes, fue premiado otra vez con el grado de Subinspector médico de segunda clase (teniente coronel) que ahora sí ostentó durante años sin permutar. En la segunda mitad del siglo XIX y a consecuencia de las constantes guerras, era frecuente casos como el de Ezequiel en el Cuerpo de Sanidad, donde a los pocos años de servicio casi todos ostentaban, a título honorífico, uno o dos grados superiores al empleo que les correspondía por antigüedad ${ }^{8}$. Ya de vuelta en Miranda del Ebro se encargó del hospital allí montado hasta que, en el mes de abril de 1875 , fue destinado al $4^{\circ}$ Regimiento de Artillería a Pie, con plaza en A Coruña.

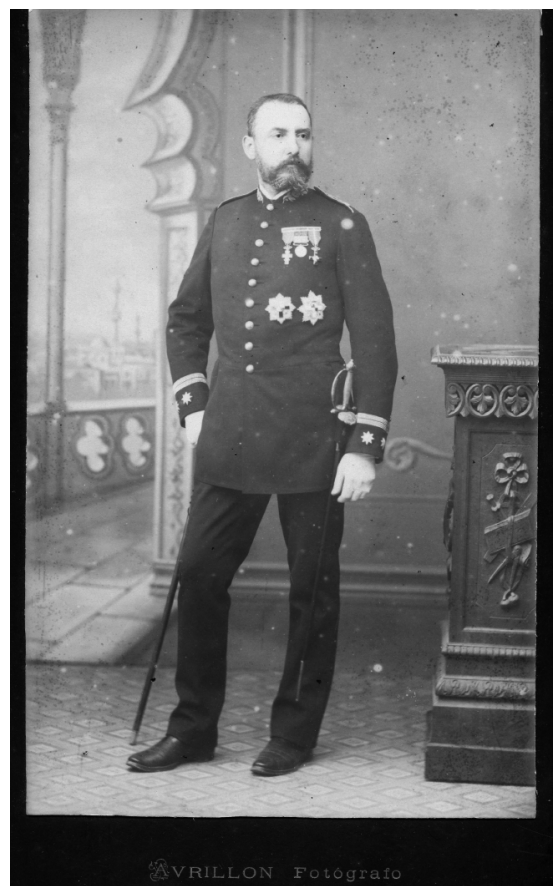

Ezequiel Abente y Lago, Subinspector médico de $2^{\mathrm{a}}$ clase (Teniente coronel). ARAG.

${ }^{8}$ MASSONS, José María, Historia de la sanidad..., 202. 
Desde entonces comienza en Coruña una nueva etapa en su vida en la que deja atrás la guerra y los hospitales de campaña, desempeñando durante años su oficio en el citado regimiento. Sin dejar el mismo y por espacio de pocos meses estuvo destinado otra vez en el Ejército del Norte, como Jefe de Sanidad militar -en comisión- de la $1^{\text {a }}$ Brigada de la VI división del $1^{\text {er }}$ Cuerpo, además de una fugaz estancia en el hospital de Santa Cruz de Tenerife. Por su contribución a lo largo de la guerra contra los carlistas, aparte de la citada condecoración por la batalla de Arrigorriaga, también recibirá la Cruz de segunda clase del Mérito Militar con distintivo rojo, la Medalla de la Guerra Civil de 1873-74 y el nombramiento de Benemérito de la Patria.

Pero su tranquila vida coruñesa se trunca en mayo de 1882, cuando muere su esposa Rafaela con tan sólo 32 años de edad ${ }^{9}$. Unos meses después, en noviembre, es promovido a Médico mayor efectivo, es decir, por antigüedad y llega su primer nombramiento como director de un hospital militar, en este caso el de Badajoz. Sin embargo, no seguirá como tal y volverá a Galicia tras casarse en diciembre de 1883 con Cristina García de la Torre, perteneciente a una pudiente familia coruñesa ${ }^{10}$. En octubre de 1884, tras pasarse casi todo el año en situación de reemplazo, se verifica su traslado al Hospital Militar de Coruña, coincidiendo con el nacimiento de su primer hijo con su segunda esposa, con la que tendrá otro hijo dos años después ${ }^{11}$.

En 1889 el ascenso al empleo de Subinspector médico de segunda clase, cuyo grado ostentaba desde hacía años, implica nuevos destinos como director de los hospitales militares de Granada y Vitoria, volviendo al de Coruña, ya como director, en febrero de 1893. En 1894 asciende a Subinspector médico de primera clase (coronel), quedando adscrito al Cuadro de Eventualidades del Servicio con residencia también en Coruña. Finalmente, en octubre de 1896, es nombrado otra vez director del Hospital Militar coruñés. El nombramiento llega en un momento crucial, en plena guerra colonial. Por ello, durante la misma, desempeñará la dirección del centro en concepto de comisión, mientras permanece destinado en la plantilla del Ministerio de Guerra y se le encarga la Inspección de Sanidad Militar del VIII Cuerpo del Ejército, es decir, del reconocimiento médico de los soldados de este cuerpo que parten a la guerra. El nuevo conflicto en las colonias de ultramar, tras la entrada en escena de los EE.UU., se convierte en un Desastre, el del 98. El 1 de mayo y el 3 de julio se suceden las derrotas navales de Filipinas y Cuba, tras lo cual se firma el armisticio de Washington del 12 de agosto. Poco antes del inminente final de la guerra con-

\footnotetext{
${ }^{9}$ ARAG, Fondo Ezequiel Abente y Lago, 33/1.

${ }^{10} \mathrm{Ibíd}$.

${ }^{11}$ Ibíd.
} 
cluye su atribución temporal y el 23 de julio Ezequiel vuelve a ser nombrado director en plantilla del Hospital Militar de Coruña. A partir de agosto empiezan a llegar al puerto de Coruña los barcos de Cuba repletos de soldados heridos y enfermos. La cantidad y el calamitoso estado en el que llegan los soldados hacinados en los barcos causó un gran impacto en la ciudad y desbordó la capacidad del Hospital Militar. En estas circunstancias es cuando Ezequiel se distingue al frente del personal sanitario del centro, en el que serán atendidos, curados y trasladados a otros hospitales muchísimos soldados, como se hace constar en su expediente militar:

"Con motivo del notable aumento de enfermería producido por la llegada de frecuentes expediciones de repatriados al puerto de La Coruña demostró, tanto en el desempeño de la Inspección de Sanidad Militar del VIII Cuerpo del Ejército como en la Dirección del Hospital Militar de dicha plaza, notable acierto en las disposiciones adoptadas que hicieron que los desembarcos se efectuasen con perfecta regularidad, los hospitalizados estuvieron siempre convenientemente asistidos y las evacuaciones sobre los hospitales del interior se realizasen en la oportunidad y con el método precisos, por todo lo cual mereció repetidos pláceres de las Autoridades superiores de la Región"12.

Dos años después, en 1900, se le concederá la Cruz de tercera clase del Mérito Militar con distintivo blanco "por los relevantes servicios prestados en la plaza de La Coruña, con motivo de la organización de tropas para ultramar y repatriación de las mismas"13. La crisis tocó muy de cerca a la familia Abente, ya que tres de sus miembros fueron testigos privilegiados de lo acontecido durante aquellos días. Mientras Ezequiel se afanaba en su difícil labor al frente del hospital, su cuñado José Valdés Díaz interrumpió su veraneo en Galicia y se marchó en septiembre del 98 a París, como miembro de la comisión encargada de firmar el finiquito del imperio colonial ${ }^{14}$. Y, a finales de ese mismo mes, su primo Eduardo Pondal envía una sentida carta a una hermana en la que nos dejó constancia del "terrible espectáculo de la repatriación de parte de nuestro ejército":

"De aquella florida juventud de que te hablé en una carta, cuando partió para la campaña, no restan ya más que cadáveres ambulantes, de tal modo que ni sus pobres madres ni sus deudos ni sus hogares los reconocerían. ¡Tan mudados vienen de aquello que fueron!"15.

${ }^{12}$ AGMS, Expedientes personales, Abente Lago, Ezequiel.

${ }^{13}$ Ibíd.

14 VALDÉS HANSEN, Felipe, El Director General de Aduanas José Valdés Díaz (1854-1916). Del Ponteceso de los Pondal a la Paz de París, Sada 2005, 53-64.

${ }^{15}$ FERREIRO, Manuel, Pondal. Do dandysmo á loucura, Santiago de Compostela 1991, 196. 
Superada la crisis humanitaria continuará en Coruña al frente del hospital, hasta que en 1901 es ascendido al empleo de Inspector médico de segunda clase y destinado como tal en la $3^{\text {a }}$ (enero), $6^{\text {a }}$ (febrero) y $8^{\text {a }}$ Región militar (1902), las dos últimas con capitanía general o sede en la ciudad de Coruña. Suprimida esta última región militar en 1904, con motivo de la reorganización del ejército, Ezequiel quedó en situación de cuartel hasta 1905. En abril de dicho año, siendo ya el primero de su escala, fue ascendido y nombrado Inspector médico de primera clase (brigadier o general de brigada) del IV Cuerpo del Ejército y, poco después, del I Cuerpo con mando y cuartel en Madrid. La relevancia y prestigio que implicaba su rango y empleo se acrecienta y refrenda, poco después, con el nombramiento como vocal del Real Consejo de Sanidad.

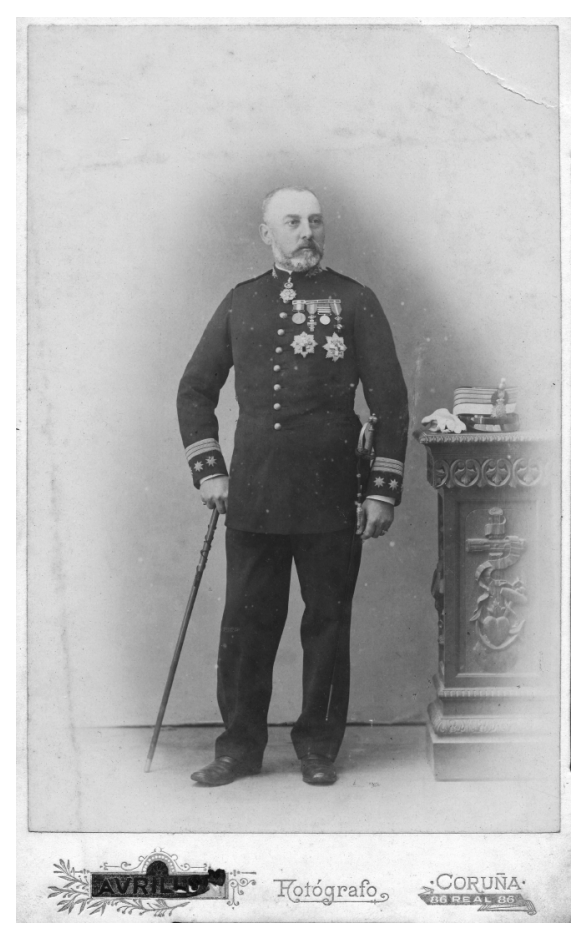

Ezequiel Abente y Lago, Subinspector médico de $1^{\text {a }}$ clase (Coronel). ARAG.

Durante dos años desempeñó el empleo más importante de su escalafón hasta que en 1907, coincidiendo con su onomástica al cumplir la edad estipulada, se verificó su cese y pase a la reserva tras casi 43 años de servicio. Desde entonces el matrimonio Abente-García de la Torre establece su residencia fija en Madrid, tras- 


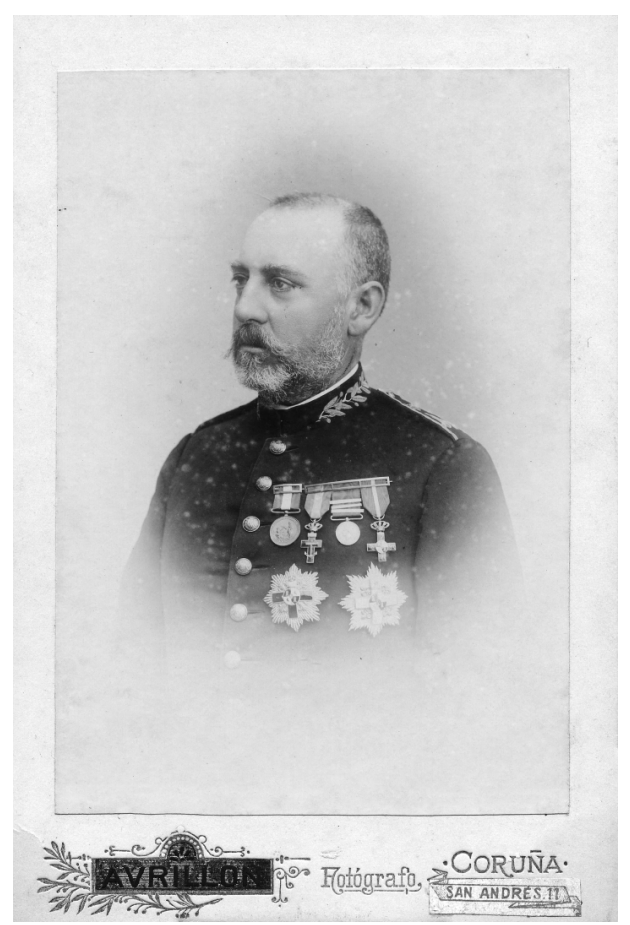

Ezequiel Abente y Lago, Subinspector médico de $1^{\mathrm{a}}$ clase (Coronel), ca. 1900. ARAG.

ladándose en los períodos vacacionales a la casa y propiedades que su mujer había heredado en Oleiros, a las afueras de la ciudad de Coruña. En 1919 sabemos que, tanto él como su mujer, no gozan de buena salud, pues en el mes de agosto solicita al arzobispo de Santiago permita oficiar misa en el oratorio de su casa los días de precepto, dada la avanzada edad de ambos y la distancia que les separaba de la iglesia parroquial ${ }^{16}$. Finalmente, Ezequiel fallecerá en su casa de Madrid el 17 de diciembre de 1923, siendo enterrado al día siguiente en el antiguo cementerio de la Sacramental de San Justo, muy cerca del río Manzanares ${ }^{17}$.

Unos años antes de morir, en 1919, había sido distinguido con la Gran Cruz, Cruz y Placa de San Hermenegildo. Como ya vimos, por sus méritos como soldado y sanitario en el campo de batalla había obtenido las cruces de primera y segunda clase del Mérito Militar con distintivo rojo, la Cruz de primera clase del Mérito Naval con igual distintivo y la Medalla de la Guerra Civil de 1873-74, aparte del

\footnotetext{
16 ARAG, Fondo Ezequiel Abente y Lago, 33/1.

17 Sin título, $A B C, 18-12-1923,33$.
} 


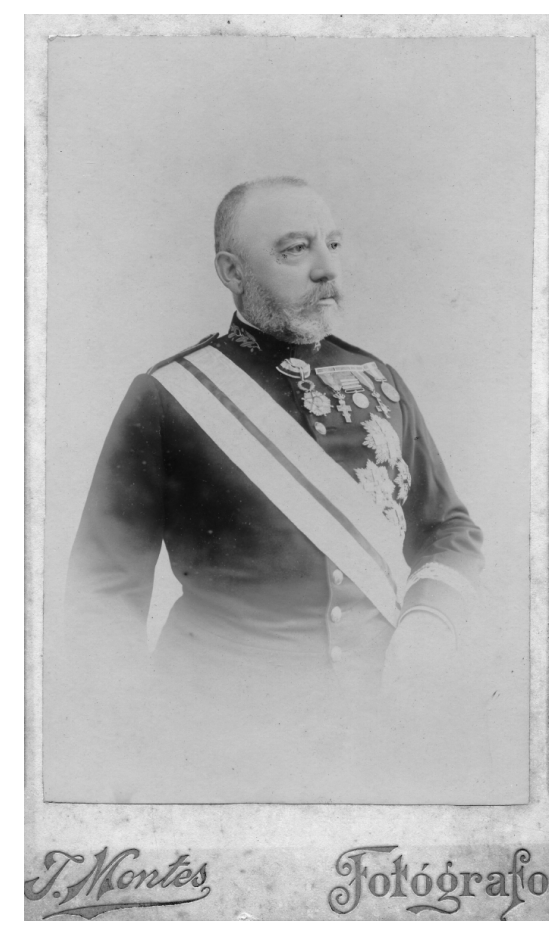

Ezequiel Abente y Lago, Inspector médico de $1^{\mathrm{a}}$ clase (General de brigada). ARAG.

nombramiento como Benemérito de la Patria. Pero, además, por sus servicios especiales se le concedió la Gran Cruz del Mérito Militar con distintivo blanco y la de tercera clase de igual color, además de la Cruz de segunda clase de la Orden Civil de Beneficencia. También fue distinguido con la Encomienda de Isabel la Católica, la Cruz de segunda clase del Mérito Militar con distintivo blanco y la medalla de Alfonso XIII con motivo de acontecimientos y celebraciones puntuales de relevancia (enlace de Alfonso XII, mayoría de edad de Alfonso XIII, etc.).

Pero el mayor reconocimiento llegó mucho tiempo después, cuando el Ejército de España entregó el Hospital Militar de A Coruña a la Xunta de Galicia en 1996. El nuevo uso civil implicó la necesidad de rebautizar el histórico centro. Honrando la memoria de nuestro biografiado y reconociendo su competente dirección, durante la repatriación de los soldados de Cuba, el más antiguo de los hospitales gallegos en servicio fue rebautizado con el nombre de Hospital Abente y Lago ${ }^{18}$.

18 "El Hospital Militar de La Coruña pasará a llamarse Abente y Lago", La Voz de Galicia, 20-91996, 1, 31 . 


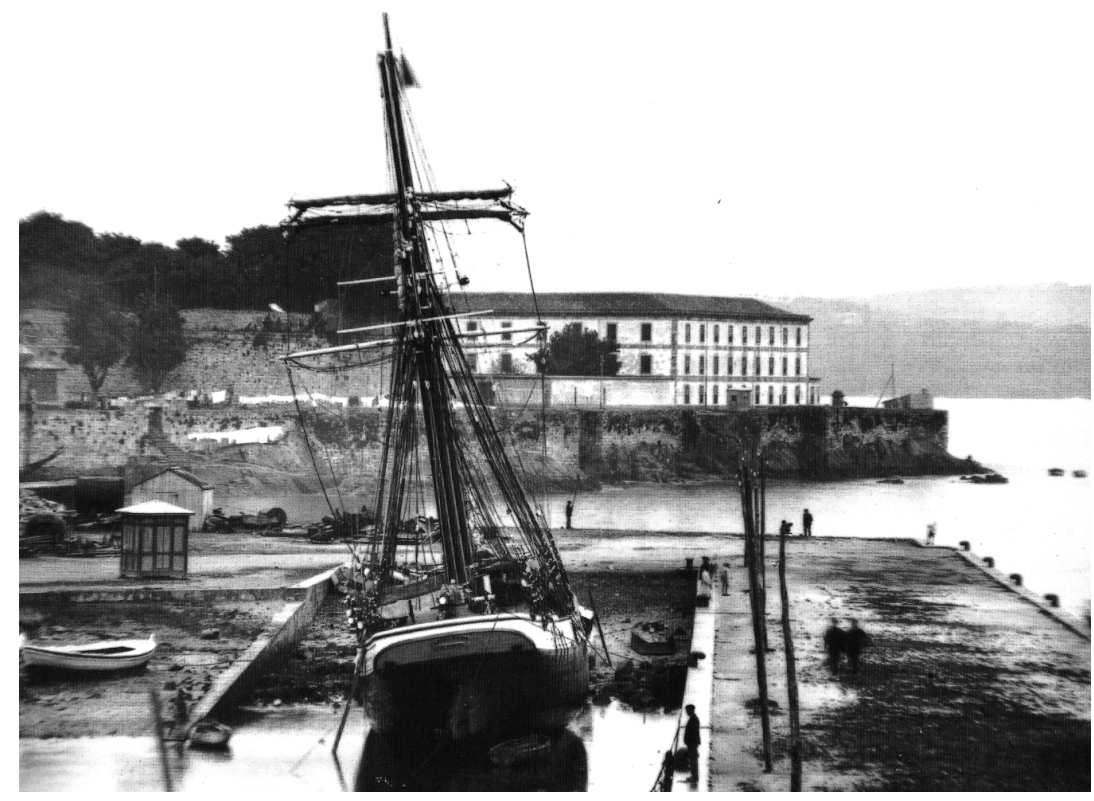

El Hospital Militar de Coruña a principios del siglo XX.

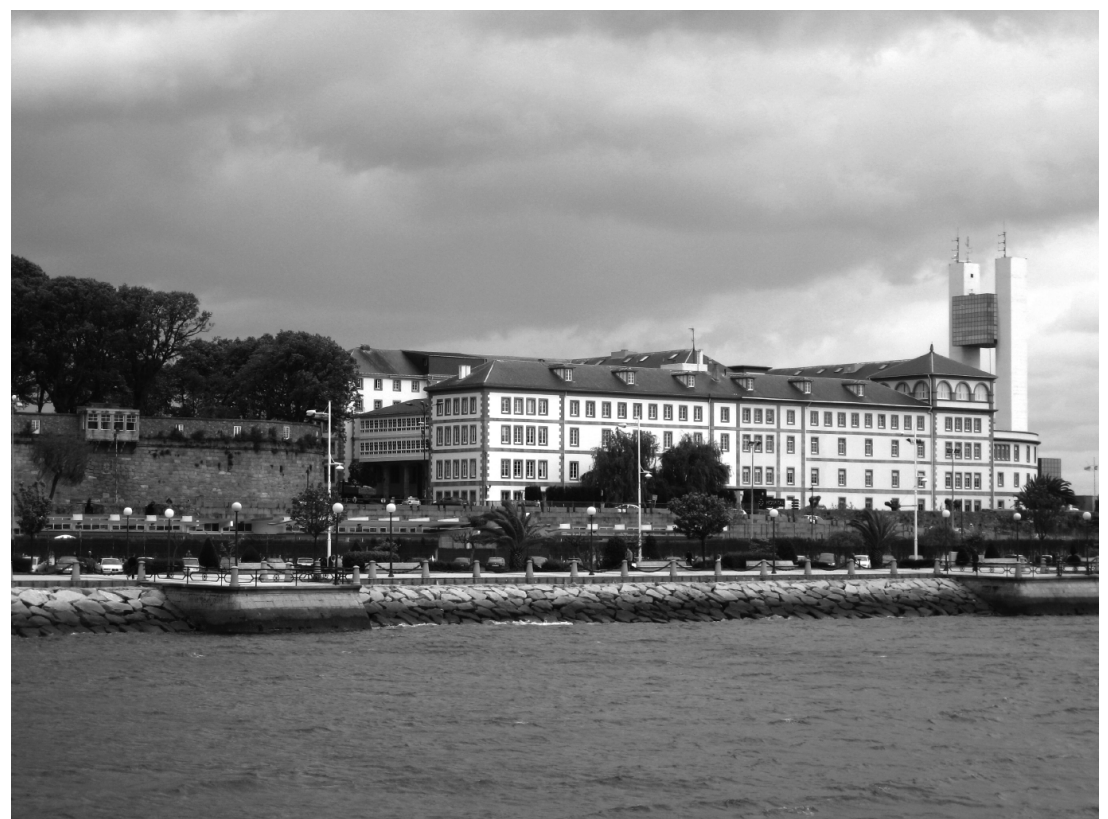

El antiguo Hospital Militar, hoy Hospital Abente y Lago (Foto F. Valdés Hansen). 


\section{Empleos de Ezequiel Abente y Lago}

\begin{tabular}{ll}
\hline FECHA & EMPLEO \\
\hline 1864 & $2^{\text {o }}$ Ayudante médico por oposición. \\
\hline 1867 & $1^{\text {er }}$ Ayudante médico por antigüedad. \\
\hline 1868 & Grado de Médico mayor por la gracia general del 68. \\
\hline 1873 & Médico mayor por mérito de guerra*. \\
\hline 1874 & Grado de Subinspector médico de $2^{\text {a }}$ clase por mérito de guerra. \\
\hline 1882 & Médico mayor por antigüedad. \\
\hline 1889 & Subinspector médico de $2^{\text {a }}$ clase por antigüedad. \\
\hline 1894 & Subinspector médico de $1^{\text {a }}$ clase por antigüedad. \\
\hline 1901 & Inspector médico de $2^{\text {a }}$ clase por antigüedad. \\
\hline 1905 & Inspector médico de $1^{\text {a }}$ clase por sus servicios. \\
\hline
\end{tabular}

* Unos meses antes, en 1872, fue premiado con el grado de Subinspector médico de $2^{\text {a }}$ clase que le fue permutado por el empleo de Médico mayor.

\section{Destinos de Ezequiel Abente y Lago}

\begin{tabular}{ll}
\hline FECHAS & DESTINO \\
\hline $1864-65$ & Batallón de Cazadores de Tarifa. \\
\hline $1865-67$ & Hospital Militar del Peñón de Vélez. \\
\hline 1867 & Batallón Cazadores de Tarifa. \\
\hline $1867-68$ & Situación de reemplazo. \\
\hline $1868-69$ & $2^{\text {a }}$ Batallón $R^{\circ}$ Infantería de Galicia. \\
\hline $1869-70$ & $2^{\circ}$ Batallón $R^{\circ}$ Infantería de Iberia. \\
\hline 1870 & $1^{\circ}$ Batallón $R^{\circ}$ Infantería de Valencia. \\
\hline $1870-72$ & $2^{\circ}$ Batallón $R^{\circ}$ Infantería de Cuenca. \\
\hline $1872-73$ & Situación de reemplazo en Muxía. \\
\hline 1873 & En comisión en el Ejército del Norte y R Infantería Almansa. \\
\hline $1873-74$ & R $^{\circ}$ Caballería de Talavera. \\
\hline $1874-75$ & $1^{\text {er } \text { Batallón } R^{\circ} \text { Ingenieros. }}$ \\
\hline 1875 & $2^{\circ}$ Batallón $4^{\circ} R^{\circ}$ de Artillería a Pie y Hospital Militar de Tenerife. \\
\hline $1875-82$ & $2^{\circ}$ Batallón $4^{\circ} R^{\circ}$ de Artillería a Pie. \\
\hline $1882-84$ & Director del Hospital Militar de Badajoz. \\
\hline 1884 & Situación de reemplazo en Coruña. \\
\hline $1884-89$ & Hospital Militar de Coruña. \\
\hline $1889-90$ & Director del Hospital Militar de Granada. \\
\hline $1890-93$ & Jefe de Servicio y Director interino del Hospital de Vitoria. \\
\hline $1893-94$ & Director del Hospital Militar de Coruña. \\
\hline $1894-96$ & Cuadro Eventual en Coruña. \\
\hline
\end{tabular}




\begin{tabular}{ll}
\hline 1896 & Director del Hospital Militar de Coruña. \\
\hline $1896-98$ & $\begin{array}{l}\text { Ministerio de Guerra, Director en comisión del Hospital Militar de } \\
\text { Coruña e Inspector de Sanidad del VIII Cuerpo del Ejército. }\end{array}$ \\
\hline $1898-01$ & Director del Hospital Militar de Coruña. \\
\hline 1901 & Inspector de Sanidad de la $3^{\text {a }}$ Región. \\
\hline $1901-02$ & Inspector de Sanidad de la $6^{\text {a }}$ Región. \\
\hline $1902-04$ & Inspector de Sanidad de la $8^{\text {a }}$ Región. \\
\hline $1904-05$ & Situación de cuartel. \\
\hline 1905 & Inspector de Sanidad del $4^{\circ}$ Cuerpo del Ejército. \\
\hline $1905-07$ & Inspector de Sanidad del $1^{\text {er }}$ Cuerpo/1 ${ }^{\text {a }}$ Región del Ejército. \\
\hline
\end{tabular}

\section{Condecoraciones, honores y distinciones de Ezequiel Abente y Lago}

\begin{tabular}{|c|c|}
\hline FECHA & CONDECORACIÓN \\
\hline 1872 & $\begin{array}{l}\text { Cruz de } 1^{\text {a }} \text { clase del Mérito Militar con distintivo rojo por la acción de } \\
\text { Arrigorriaga, durante la guerra carlista ( } 20 \text { jul). }\end{array}$ \\
\hline 1876 & $\begin{array}{l}\text { Benemérito de la patria por haber contribuido a la victoria contra los } \\
\text { carlistas ( } 3 \text { jul.). }\end{array}$ \\
\hline 1876 & $\begin{array}{l}\text { Cruz de } 2^{\text {a }} \text { clase del Mérito Militar con distintivo blanco por los } \\
\text { servicios especiales en sus destinos ( } 12 \text { ago.). }\end{array}$ \\
\hline 1876 & $\begin{array}{l}\text { Cruz de } 2^{\text {a }} \text { clase del Mérito Militar con distintivo rojo por servicios de } \\
\text { guerra, en lugar de la de servicios especiales del } 12 \text { de agosto de } 1876 \\
(9 \text { nov.). }\end{array}$ \\
\hline 1877 & Medalla de la Guerra Civil de 1873-74 (25 mar.). \\
\hline 1878 & $\begin{array}{l}\text { Encomienda de Isabel la Católica por el enlace real de Alfonso XII } \\
\text { con María de las Mercedes ( } 22 \text { ene.). }\end{array}$ \\
\hline 1878 & $\begin{array}{l}\text { Cruz de } 1^{\text {a }} \text { clase del Mérito Naval con distintivo rojo por ayudar a } \\
\text { sofocar la insurrección republicana del Ferrol de } 1872 \text { (4 may.). }\end{array}$ \\
\hline 1880 & $\begin{array}{l}\text { Cruz de } 2^{\text {a }} \text { clase del Mérito Militar con distintivo blanco por el } \\
\text { nacimiento de la infanta María de las Mercedes }(9 \text { oct.). }\end{array}$ \\
\hline 1883 & Mención honorífica por una memoria médica (22 oct.). \\
\hline ca. 1900 & le la Orden Civil de Beneficencia. \\
\hline 1900 & $\begin{array}{l}\text { Cruz de } 3^{\text {a }} \text { clase del Mérito Militar con distintivo blanco por su labor } \\
\text { durante la repatriación de los soldados de Cuba }(20 \text { ago.). }\end{array}$ \\
\hline 1902 & $\begin{array}{l}\text { Gran Cruz del Mérito Militar con distintivo blanco por servicios } \\
\text { especiales (16 may.). }\end{array}$ \\
\hline 1902 & Medalla de Alfonso XIII por la mayoría de edad del rey (19 jul.). \\
\hline 1919 & Gran Cruz, Cruz y Placa de San Hermenegildo (10-15 ene.). \\
\hline
\end{tabular}




\title{
ASCENDENCIA ABENTE DE EZEQUIEL ABENTE Y LAGO
}

\author{
ANDRÉS DE ABENTE Y COSTOYA \\ +1748 (Allones) \\ CC \\ ÁNGELA VARELA RODRIGUEZ \\ $1708 /+1762$ (Allones) \\ 1 \\ IGNACIO ABENTE VARELA \\ 1736 (Allones) $/+1802$ (Laxe) \\ Administrador de las rentas reales de Laxe \\ CC \\ Ma JOSEFA VALES Y ÁLVAREZ DE CALO \\ 1749 (Laxe) / † 1814 (Laxe) \\ ISIDRO ABENTE VALES \\ 1772 (Laxe) $/ † 1842$ (Laxe) \\ Administrador de las rentas reales y alfolíes de Laxe \\ cC \\ $M^{a}$ ROSARIO CHANS MOURELLE \\ (Corme) / + 1847 (Laxe)<smiles>C=[IH]</smiles> \\ LEANDRO ABENTE CHANS \\ 1809 (Laxe) / † 1896 (Muxía) \\ Médico y cirujano en Muxía \\ cC \\ MANUELA LAGO DÍAZ \\ 1819 (Muxía) / † 1900 (Muxía) \\ EZEQUIEL ABENTE Y LAGO \\ 1841 (Corcubión) / † 1923 (Madrid) \\ General del Cuerpo de Sanidad Militar \\ $\mathrm{CC}$ \\ RAFAELA ESCOBAR LÓPEZ / \\ CRISTINA GARCÍA DE LA TORRE
}

\section{BIBLIOGRAFIA}

ANÓNIMO, Álbum-escalafón del Cuerpo de Sanidad Militar del Ejército Español con los retratos y situación de sus generales, jefes y oficiales en $1^{\circ}$ de enero de 1900, siendo Ministro de la Guerra el Excmo. Sr. Teniente General D. Marcelo de Azcárraga y Palmero y Jefe de la Sección de Sanidad Militar el Excmo. Sr. Inspector Médico de $2^{a}$ clase D. Bernardino Gallego Saceda, Madrid 1900 .

FERREIRO, Manuel, Pondal. Do dandysmo á loucura, Santiago de Compostela 1991. 
MASSONS, José María, Historia de la sanidad militar española 4, Barcelona 1994.

VALDÉS HANSEN, Felipe, El Director General de Aduanas José Valdés Díaz (1854-1916). Del Ponteceso de los Pondal a la Paz de París, Sada 2005.

\section{FUENTES IMPRESAS}

“El Hospital Militar de La Coruña pasará a llamarse Abente y Lago", La Voz de Galicia, 20-9-1996, 1,31 .

Gaceta de Madrid, 3-1-1901, 25-6; 14-2-1901, 658; 21-12-1902, 1048; 9-4-1905, 113-4; 16-12-1905, 931; 4-4-1907, 46.

Sin título, $A B C, 18-12-1923,33$.

\section{FUENTES MAMUSCRITAS}

Archivo General Militar de Segovia (AGMS), Expedientes personales, Ezequiel Abente Lago.

Archivo Histórico Universitario de Santiago de Compostela (AHUS), Universidad, Expedientes personales, leg. $5, \mathrm{n}^{\circ} .14$ y 17.

Archivo de la Real Academia Galega (ARAG), Fondo Ezequiel Abente y Lago.

PARGA PONDAL, Isidro, Genealogía ascendente de Don Isidro Pondal Abente y de su esposa Doña Teresa Araoz Royo, Laxe 1980.

\section{INTERNET}

Página del BOE. Base de datos Gazeta [http://www.boe.es/g/es/bases_datos/gazeta.php]. 